\title{
Téoros
}

Revue de recherche en tourisme

\section{Congrès et réunions d'affaires}

\section{Les tendances à surveiller}

\section{Claude Péloquin}

Volume 24, numéro 3, automne 2005

URI : https://id.erudit.org/iderudit/1071081ar

DOI : https://doi.org/10.7202/1071081ar

Aller au sommaire du numéro

Éditeur(s)

Université du Québec à Montréal

ISSN

0712-8657 (imprimé)

1923-2705 (numérique)

Découvrir la revue

Citer cette note

Péloquin, C. (2005). Congrès et réunions d'affaires : les tendances à surveiller. Téoros, 24(3), 55-57. https://doi.org/10.7202/1071081ar

Ce document est protégé par la loi sur le droit d'auteur. L'utilisation des services d'Érudit (y compris la reproduction) est assujettie à sa politique d'utilisation que vous pouvez consulter en ligne.

https://apropos.erudit.org/fr/usagers/politique-dutilisation/
Cet article est diffusé et préservé par Érudit.

Érudit est un consortium interuniversitaire sans but lucratif composé de l’Université de Montréal, l’Université Laval et l'Université du Québec à Montréal. Il a pour mission la promotion et la valorisation de la recherche. https://www.erudit.org/fr/ 


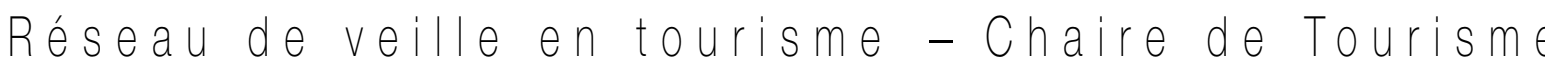 \\ Congrès et réunions d'affaires \\ Les tendances à surveiller}

\section{Claude Péloquin}

L'industrie des congrès et des réunions d'affaires est cyclique et de nature à subir l'influence du contexte économique. Après un ralentissement marqué durant les années post-11 septembre 2001, la Travel Industry Association s'attend à ce que la croissance des dernières décennies reprenne progressivement son cours pour atteindre environ 3,6\% en 2005 aux États-Unisi .

Les intervenants du secteur des congrès doivent toutefois tenir compte des grandes tendances qui exerceront une influence marquante au cours des années à venir. Deux chercheurs universitaires (Weber et Ladkin, 2004 : 47-63) ont mené une étude qualitative auprès d'un panel de 26 experts britanniques et australiens - provenant d'organisations touristiques, d'offices de congrès, d'entreprises spécialisées dans la planification de congrès, d'associations sectorielles, d'universités et de centres de congrès - afin de déterminer les consensus sur les tendances à surveiller. Celles-ci se répartissent en trois catégories : l'environnement d'affaires, la technologie ainsi que les contextes social et politique.

\section{Concurrence, préoccupation d'affaires primaire}

Un consensus apparaît (Weber et Ladkin, 2004 : 54) quant à l'importance du contexte concurrentiel global qui s'intensifiera compte tenu de la multiplication des nouveaux centres de congrès, de la modernisation des services, de la compétitivité des prix des destinations émergentes et de l'accroissement des attentes de la clientèle. Au Royaume-Uni, par exemple, les espaces pour accueillir les réunions et les congrès ne se limitent plus aux grandes villes, mais se retrouvent également dans des hôtels campagnards, dans des établissements d'éducation ou d'autres lieux d'hébergement alternatifs comme des châteaux ou certains sites historiques. Même les complexes de cinéma courtisent les événements corporatifs avec des salles d'équipement à la fine pointe de la technologie et un service clé en main.

Le panel d'experts a aussi identifié la fluctuation des devises comme étant une préoccupation majeure. Pour les destinations qui doivent composer avec une monnaie forte, l'impact peut devenir significatif dans un contexte de concurrence globale. À ce chapitre, l'appréciation considérable du dollar canadien observée au cours des derniers mois pourrait nuire à la performance du Québec sur le marché international.

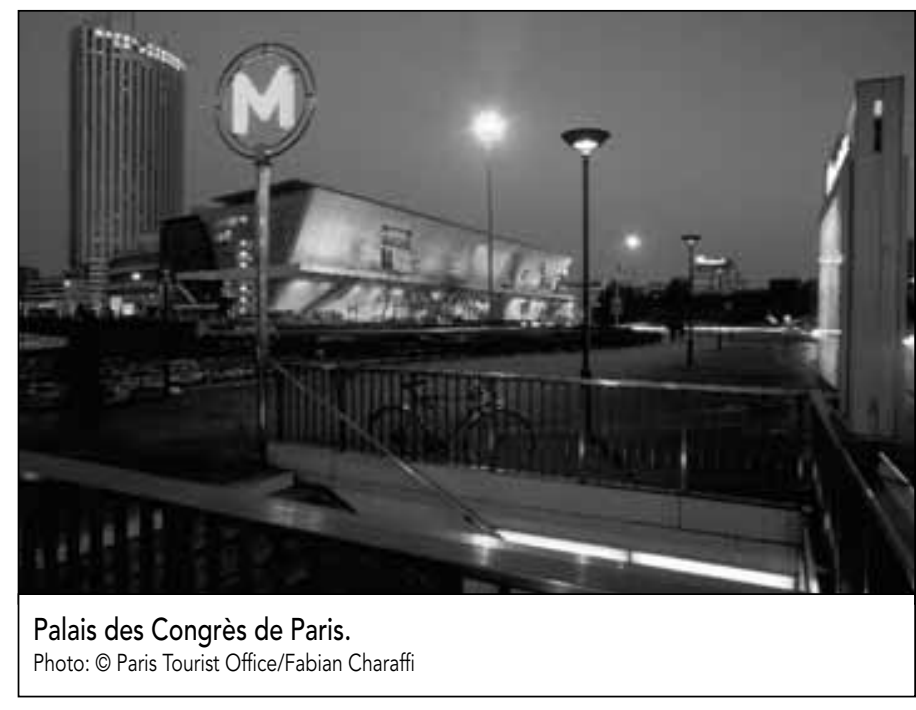

Par ailleurs, les organisations de congrès devront s'adapter à de nouvelles réalités quant aux conférences elles-mêmes. Ces dernières dureront moins longtemps et accueilleront moins de délégués. De tels changements se traduiront sur le plan marketing par le développement de relations à long terme avec la clientèle afin d'assurer une certaine stabilité et de favoriser le repeat business.

Déjà identifiés en 2003 par Meeting Professionals International comme les principaux facteurs technologiques d'influence sur la planification, les délais de réservation plus courts résultant principalement d'une efficacité accrue des échanges d'information et des services de réservation en ligne jouent un rôle direct sur la capacité d'organiser les événements plus rapidement ${ }^{2}$. Cela ajoute inévitablement de l'incertitude quant à la planification à long terme du calendrier d'événements.

\section{Avancées technologiques}

L'utilisation de nouvelles technologies dans des lieux de congrès permet normalement d'accroître l'efficacité, mais elle peut aussi engendrer certains problèmes. Souvent, l'adoption des dernières avancées techniques ne s'accompagne pas d'un support approprié et, lorsque des ennuis se pointent durant les événements, l'efficience de l'organisation en souffre. Cela explique une certaine résistance à installer la technologie de pointe qui exige, de surcroit, de dispenser une formation spécialisée aux employés du centre de congrès afin qu'ils soient en mesure d'offrir un service de qualité. 
En contrepartie, la clientèle exige de plus en plus d'équipements dernier cri. Malheureusement, les coûts requis pour l'installation et la mise à jour constante de cette nouvelle technologie s'avèrent trop élevés pour les centres de congrès de moindre envergure. Sur le plan technologique, on s'attend donc à ce que les centres de congrès de plus grande envergure disposent d'un avantage concurrentiel de plus en plus important.

Depuis plusieurs années, il est régulièrement question de l'influence de la vidéoconférence sur les déplacements d'affaires. Certains experts parlent d'un véritable produit de substitution aux voyages (Denstadli et Julsrud, 2003 : 1). Force est d'admettre que certaines révisions s'imposent. Aujourd'hui, il existe un consensus généralisé (Weber et Ladkin, 2004 : 57) parmi le panel d'experts à l'effet que ces solutions Web ne remplaceront jamais le traditionnel face-à-face. Peu importe l'avancée technologique, le contact humain demeure l'ultime moyen de communication. Ainsi, les réunions d'affaires et les congrès deviennent des lieux privilégiés pour établir des contacts professionnels directs, souvent déficients lors des échanges électroniques.

\section{Tendances sociales et politiques}

Une croissance soutenue du tourisme international favorisera la demande pour le tourisme de congrès. Cela s'explique simplement par le fait que plus les gens voyagent en général, par agrément ou par affaires, plus l'idée d'assister à une conférence à l'étranger devient acceptable.

Au cours des dernières années, les habitudes de travail ont changé, un nombre grandissant de gens effectuant du travail à domicile. Ce contexte professionnel d'isolement crée une situation encore plus propice aux regroupements d'affaires qui permettent de rassembler les travailleurs dispersés.

Les changements socio-démographiques pourraient aussi engendrer de nouvelles occasions d'affaires. D'une part, avec le vieillissement de la population, un nombre important d'anciens travailleurs demeurent actifs en se joignant à diverses associations de retraités et prennent part à des réunions d'affaires ou à des congrès. D'autre part, les gens cherchent de plus en plus à équilibrer leur vie professionnelle et leur vie personnelle. Un nombre croissant de délégués choisiront d'inclure la famille en joignant un volet d'agrément à leur voyage d'affaires. Les destinations perçues comme «accueillantes» pour la famille pourraient en tirer profit.

Sur le plan politique, il est clair que la stabilité d'une destination demeure un gage de sécurité indispensable, voire un atout concurrentiel non négligeable. À ce chapitre, le Québec doit continuer de jouer la carte d'un contexte parfaitement sécuritaire, même au cœur des grandes villes. Reste à voir quels sont les autres facteurs qui influencent les planificateurs de congrès dans le choix d'une destination.

\section{L'importance d'une vitalité urbaine}

Premièrement, la vitalité du centre-ville joue un rôle primordial dans sa capacité à attirer des événements. Selon David C. Petersen, président de Town Planning Research, le dynamisme d'une ville de congrès s'évalue notamment en fonction de la variété, de la densité et de la proximité de types spécifiques de bâtiments et d'activités au centre-ville (ex. : bureaux, commerces et résidences) (2004 : 145-157).

Des chercheurs se sont aussi intéressés à quantifier ces « signes vitaux » qui rendent un centre-ville attrayant aux yeux des investisseurs et des organisateurs de congrès. On dénote cinq attributs déterminants :

- un centre-ville hétérogène,

- un environnement sécuritaire,

- un transport en commun accessible,

- une zone résidentielle abordable et attrayante, des centres commerciaux à proximité.

Les chercheurs ont effectué une analyse de 26 grandes villes américaines afin de comparer celles qui affichent les meilleurs « signes vitaux » avec celles qui présentent les meilleures performances comme ville de congrès en termes de nombre de délégués. Résultat: les deux classements se sont révélés pratiquement identiques.

Les retombées économiques résultant des dépenses des délégués sont d'ailleurs directement associées à cet indice d' " attractivité " de la ville de congrès. Une destination prisée entraînera des répercussions positives sur l'ampleur des dépenses des participants, sur le nombre de personnes qui accompagnent les congressistes et sur la durée du séjour.

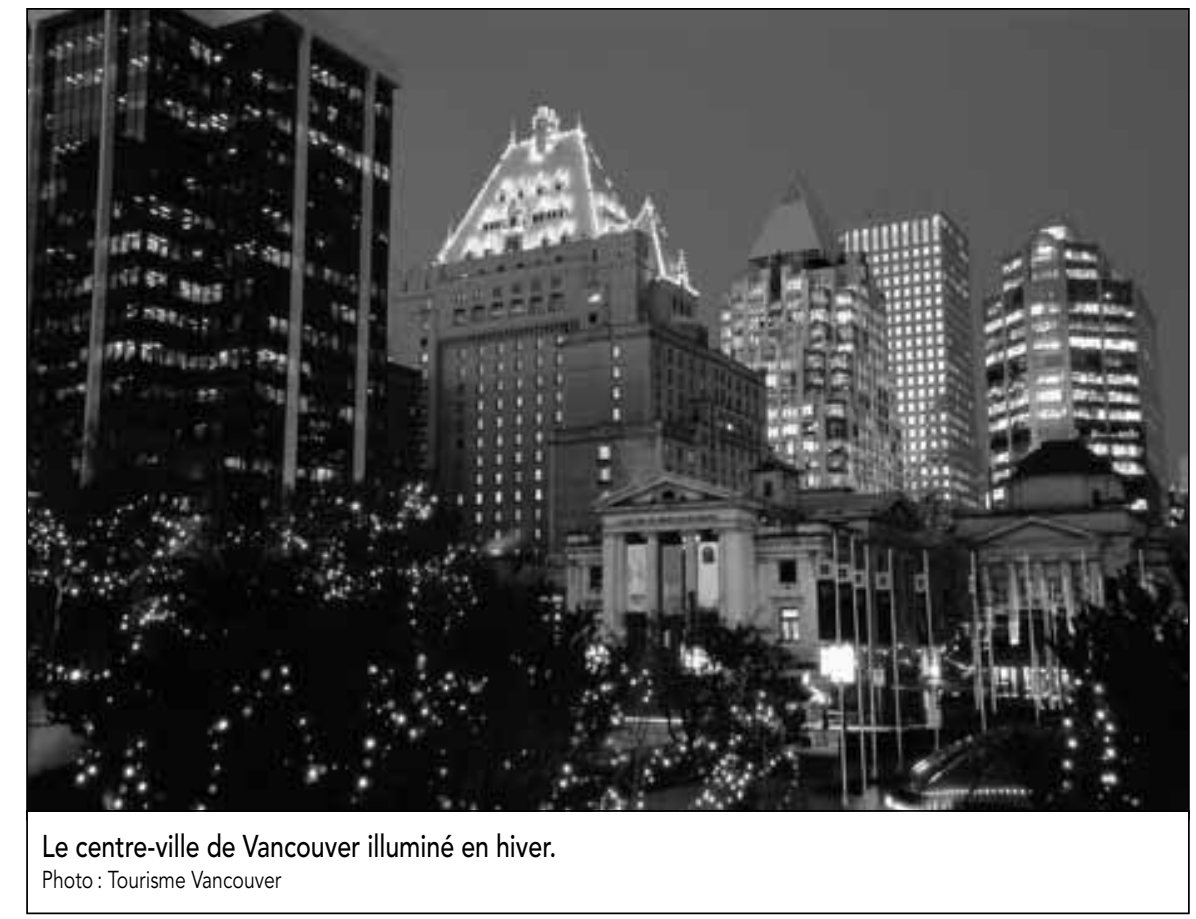


À ce chapitre, soulignons la performance de la ville de Québec et de son Centre des congrès de Québec qui, selon un sondage mené par une université allemande (Cloutier, 2005 : 4), a été reconnue comme l'une des trois meilleures destinations de congrès au monde (à égalité avec Sofia, derrière Bilbao). En 2003, Montréal figurait en deuxième position en Amérique du Nord et en quatorzième sur la scène internationale.

\section{Identifier les aspects qui font la différence}

Plusieurs aspects figurent sur la liste d'épicerie des planificateurs de congrès durant le processus de sélection d'une destination. Une étude menée par l'Université Dongguk de la Corée du Sud (Choi, 2004 : 65-80) visait à identifier les facteurs les plus susceptibles de procurer une expérience d'ensemble positive et, surtout, les raisons pour lesquelles les organisateurs choisiront de retenir à nouveau cette destination de congrès. L'enquête a été réalisée auprès de plus de 250 associations responsables de l'organisation de congrès.

Voici les huit facteurs retenus pour l'étude:

1. Le prix (comprend plusieurs aspects tels que le tarif des salles, des chambres et du service de restauration, les espaces de réunions en gracieuseté, etc.).

2. La qualité des chambres (grandeur, apparence, confort, suppléments, etc.).

3. Le personnel de l'hôtel (amabilité, efficacité, facturation adéquate, résolution de problèmes, etc.).

4. Le caractère abordable de la destination dans son ensemble (activités et attraits touristiques, accueil des résidents et du personnel de l'Office de tourisme et de congrès, etc.).

5. La qualité des salles de réunions et de conférences (éclairage, ambiance, environnement sonore, disposition des salles, disponibilité de l'équipement audiovisuel, confort des sièges, etc.).

6. L'inventaire (nombre de salles de réunions, capacité des salles et du stationnement, espace banquet, nombre de chambres disponibles, etc.).

7. La localisation (la proximité des centres commerciaux, des attraits, du quartier des affaires, des restaurants, des équipements de loisirs et la distance à parcourir pour les délégués).

8. La qualité des services complémentaires (disponibilité, sur le site de l'événement, d'un centre d'affaires et d'infrastructures de loisirs).

\section{Ce qui compte vraiment}

Parmi ces huit facteurs, seulement trois ont exercé une influence statistiquement significative sur la satisfaction globale de l'expérience, soit :

- la qualité des salles de réunions,

- le personnel de l'hôtel,

- la qualité des chambres.

L'étude coréenne pousse la démarche un peu plus loin en évaluant si l'un de ces trois facteurs affichait une corrélation significative et positive auprès des répondants sur leur intention de revenir à la même destination de congrès. Seule la variable de la qualité des salles de réunions et de conférences obéit à cette condition.
On peut certes conclure qu'il vaut la peine de maintenir un niveau d'investissement adéquat afin de compter sur des salles dédiées aux événements qui sauront combler les attentes des clients les plus exigeants. Pour plusieurs, c'est la composante de l'appréciation globale qui permettra véritablement de créer une expérience positive et qui favorisera le repeat business. Sur le plan marketing, il est aussi important de bien mettre en valeur les meilleurs atouts du centre-ville et de faire la démonstration d'un dynamisme urbain propice à l'accueil de congrès.

Claude Péloquin est analyste au Réseau de veille en tourisme de la Chaire de tourisme, École des sciences de la gestion (Université du Québec à Montréal).

\section{Notes}

1 [http://www.hospitalitynet.org/news/4021099.search?query=tia+3], consulté le 2 septembre 2005.

2 [http://www.mpiweb.org/media/home/futurewatch.pdf], consulté le 2 septembre 2005.

\section{Bibliographie}

Biarritz, Anne (2005), « Organiser un événement corporatif dans une salle de cinéma ", Le Planificateur, mai.

Choi, Jeong-Ja (2004), « Factors Influencing State Association Planners' Overall Satisfaction with a Convention Experience ", Journal of Convention \& Event Tourism, vol. 6, $\mathrm{n}^{\circ} 4$.

Cloutier, Patricia (2005), « Le Centre des congrès parmi les trois meilleurs au monde ", Le Soleil, 22 juillet, p. A4.

Denstadli, Jon Martin, et Tom Erik Julsrud (2003), «Videoconferencing: Increased Use, Less Travel? », Institute of Transport Economics, Nordic Road \& Transport Research, n 3.

Hospitality Net (2004), «Travel Industry Optimistic for 2005 », [http://www.hospitalitynet.org/news/4021099.search?query=tia+3], 2 novembre.

Jolicœur, Martin (2005), "Le Palais des congrès va jusqu'à offrir gratuitement ses salles ", Les Affaires, 7 mai.

Kouchner, Martin (2005), «Touristes en cravate », Commerce, vol. 106, nº 1, janvier.

Meeting Professionals International (2003), « Welcome to Futurewatch 2003 », MPI, [www.mpiweb.org].

Petersen, David C. (2004), "The City as a Destination: Measuring Its Attractiveness ", Journal of Convention \& Event Tourism, vol. 6, no 1.

Priporas, Vasilios (2005), «Is It Difficult to Market a City as a Convention Destination? The Case of Thessaloniki », Journal of Convention \& Event Tourism, vol. $7, n^{\circ} 2$.

Travel Industry Association (2004), "Travel Industry Optimistic For 2005 ", Hospitality Net, [www.hospitalitynet.org], 2 novembre.

Weber, Karin, et Adele Ladkin (2004), "Trends Affecting the Convention Industry in the $21^{\text {st }}$ Century ", Journal of Convention \& Event Tourism, vol. $6, n^{\circ} 4$. 